\title{
Performance Analysis of Multimedia Traffic over MPLS Communication Networks with Traffic Engineering
}

\author{
Dr. Abdul-Bary R. Sulaiman ${ }^{1}$ and Omar Kh. Salih Alhafidh ${ }^{2}$ \\ ${ }^{1}$ College of Electronic Engineering, Computer and Information Dep. University of Mosul, Iraq \\ ${ }^{2}$ College of Computer Science and Mathematics, Computer Science Dept. University of Mosul, Iraq \\ E-mail:.'suleimana52@uomosul.edu.iq, ${ }^{2}$ Omar.kh.Alhafidh@gmail.com
}

\begin{abstract}
The recent evolutions of IP networks are seeing IP applications becoming more complex and requiring higher bandwidth consumption. More recently, IP networks are employing MPLS which is a technique can be used to improve the performance of IP networks. By use of MPLS, data packets can be switched on the basis of labels rather than routed on the basis of destination address. MPLS supports different features like traffic engineering (TE), QoS and VPNs etc. The key feature of MPLS is its TE, which plays a vital role for minimizing the congestion by efficient load balancing and management of the network resources. Due to lower network delay, efficient forwarding mechanism, enhancing the speed of packet transfer, scalability and predictable performance of the services provided by MPLS technology makes it more suitable for implementing real-time applications such as VoIP and video Conferencing. This paper evaluated the performance measures such as delay variation, delay, page response time, throughput, and packet drop for different types of traffic (voice, video, data) in their movement in a congested network for both MPLS-TE and conventional IP network. OPNET modeler is used to simulate the both networks, the simulation study is conducted in this paper; It is a means to illustrate the benefits of using MPLS-TE for multimedia applications.
\end{abstract}

Keywords: Multiprotocol Label Switching (MPLS), Traffic Engineering (TE), Voice Over IP (VoIP), Internet Protocol (IP), Multiprotocol Label Switching Traffic Engineering (MPLS-TE), OPNET.

\section{INTRODUCTION}

Lately the Internet provide us with real-time applications which need to have the smallest possible end-to-end delay. Such applications include voice and video conferencing. These applications are bandwidth demanding and most of the times, a new higher capacity link is needed in order to provide the required delays, something that it is not cost effective. Therefore, a new way is needed in order to run these applications and still maintain the low end-to-end delay without having to spent more money on upgrading the network.

In interactive applications of real time sound transmission, the overall one way delay needs to be short in order to give the user an impression of real time responses. A maximum value on the order of 0.1 to 0.5 seconds is required to accomplish this goal. The International Telecommunication Unit (ITU) G.114 specification recommends less than a $150 \mathrm{~ms}$ one-way end-to-end delay for high-quality real time traffic and acceptable between 150$400 \mathrm{~ms}$. And for video application, a video stream should not exceed 250ms [1][23][24]. The best effort protocols cannot guarantee such limits because the datagrams do not follow a fixed path and may arrive at the destination out of order. With increased congestion, the queues get longer resulting in increased jitter. These problems make conventional IP networks unsuitable for connection-oriented applications such as real-time applications. MPLS has emerged as the key integration technology for carrying voice, video, and data traffic over the same network, and it is technology which plays an important role in the next generation networks by providing Quality of 
Service (QoS) and TE (traffic engineering). In an MPLS network, LSPs (Label Switched Path) are installed from an ingress node to an egress node prior to start of transmission. Each LSP can be specified with features that include time constraints and reliability. Therefore, the connection-oriented applications can take advantage of the "virtual connections" set by MPLS that satisfy some constraints. Since the LSPs are stackable, traffic from different flows sharing some common characteristics can be aggregated on an LSP. These characteristics may include common egress and identical QoS and protection requirements [2][3].

Previous works focus on performance comparison of network traffic (without considering video and VoIP traffic) between both MPLS and non-MPLS networks by simulation. The related work for comparing the real-time voice communications between MPLS and non-MPLS had done either through analytical models or from theoretical analysis [2],[3], [4], [5], and [6]. $\operatorname{In[7][8]~the~authors~}$ mainly focuses on comparative analysis of MPLS over non-MPLS networks and different type of LDP (Label Distribution Protocol) are discussed with respect to TE.

Our contribution is to design a network model using OPNET modeler for comparing VoIP and video Conferencing traffic performance in addition to normal data FTP(File Transfer Protocol) on both MPLS and non-MPLS networks.

\subsection{Traditional IP Routing}

Internet Protocol (IP) allows a global network among an endless mixture of systems and transmission media [2][7].The IP was created as a connectionless network layer protocol that makes no attempt to discriminate between various application types [6][9]. The main function of IP is to send the data from the source to destination. Data is constructed as a series of packets. All the packets are routed through a chain of routers and multiple networks to reach the destination. In the Internet, router takes independent decision on each incoming packet. When a packet arrives at a router, the router has to consult its routing table to find the next hop for that packet based on the packets destination address in the packets IP header (longest match prefix lookup), as explained in Fig. 1 [2][10][11]. To build routing tables each router runs IP routing protocols like Border Gateway Protocol (BGP), Open Shortest Path First (OSPF) or Intermediate System-to-Intermediate System (IS-IS). When a packet traverses through the network, each router performs the same steps of finding the next hop for the packet until it reach the destination [7][12]. As a result traffic is concentrate across a smaller number of optimized data paths to the detriment of other links, which frequently remain underutilized. All arriving data flows on various ingress interfaces on the same node that are bound for the same destination are always consolidated across a common path. The compounded effect of concentrating large data flows across a small number of links often produces traffic bottlenecks. Even in the face of congested links, traditional routing protocols will continue to forward traffic across these same paths until packets are dropped. To accommodate highly interactive application flows with low delay and packet loss thresholds, there is a clear need to more efficiently utilize the available network resources. The process whereby this is accomplished is known as traffic engineering and MPLS provides these capabilities [6].

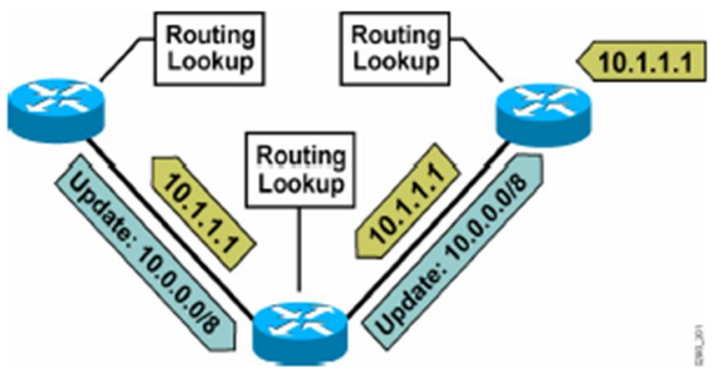

Fig. 1 Traditional IP routing

\section{MPLS}

MPLS (Multi-Protocol Label Switching), as standardized by the Internet Engineering Task Force (IETF) is a layer 3 packet switching technology that transmits traffic effectively and supports QoS on the Internet. It is expected that MPLS improves the performance of routing in the network layer [13][14].

MPLS is used in Internet Service Provider (ISP) networks and as a backbone to Internet Protocol (IP) to provide guaranteed efficient bandwidth and Quality of Service (QoS) provisioning in the network [4][5][15]. MPLS supports multiple Layer 2 protocols such as ATM, Frame Relay and Ethernet. Because of the variety of the underlay network structures, MPLS is able to establish endto-end IP connections with different QoS characteristics associated with the multiple transport media [16], its objective to give the router a big power of communication [4]. So it bases especially on a label (number) inserted between the layer 2(data link layer) and the layer 3(network layer) in the OSI model as in Fig. 2; therefore it is called layer 2.5 protocol [2] [4]. 


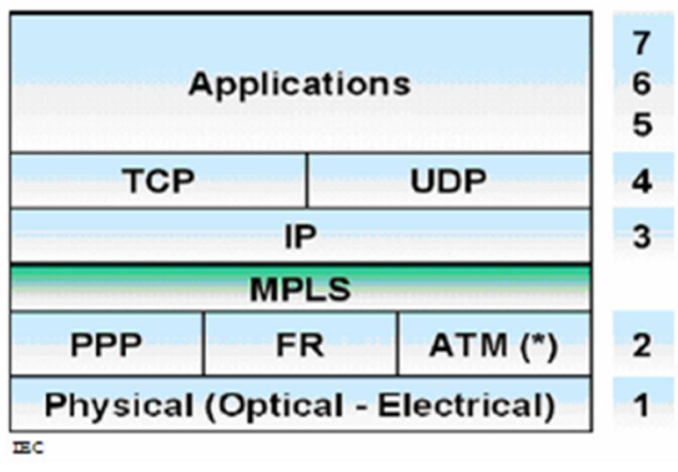

Fig. 2 OSI reference model for MPLS

In a MPLS network, incoming packets are assigned a "label" by a "LER (label edge router)" according to their forwarding equivalence class (FEC). Packets are forwarded along a "label switch path (LSP)" where each "LSR (label switch router)" makes forwarding decisions based solely on the contents of the label, eliminating the need to look for its IP address so that the intermediate router does not have to perform a time-consuming routing lookup as in Fig. 3 [11]. At each hop, the LSR takes off the existing label and applies a new label for the next hop. Next hop also decides how to forward the packet by reading just the label on the packet. These established paths, Label Switch Paths (LSPs) can guarantee a certain level of performance, to route around network congestion, or to create IP tunnels for network-based virtual private networks [19].

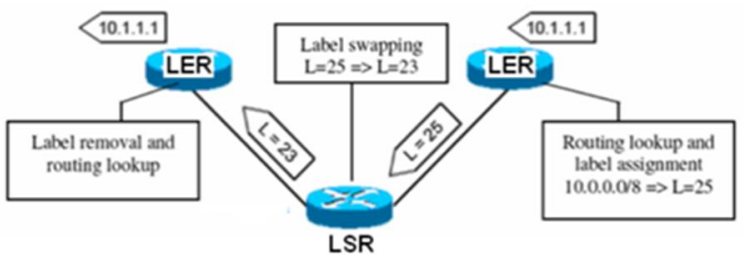

Fig.3 Example on MPLS Forwarding

\subsection{MPLS Shim Header}

Data packets when reaches the LER, "Shim Header" is placed in between layer 2 and 3 of the OSI model. This MPLS Shim Header which is structured into four parts has a total length of 32 bits; 20 bits for Label, 3 bits for Experimental (EXP), 1 bit for Bottom of Stack and 8 bits for Time to Live (TTL) which is shown in Fig. 4.

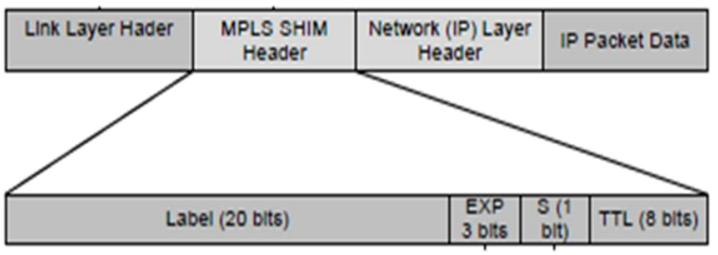

Fig. 4 MPLS Shim Header

As shown in Fig. 4, the MPLS Shim Header consists of an identifier called "Label". It acts as an identifier of Forwarding Equivalence Class (FEC), and also used for determining the Label Switched Path (LSP). Followed by Label is Experimental field (EXP) which is reserved for the experimental use or are often used for QoS purpose. Stack field (S) is used for indicating whether the label is in the bottom of Stack. If the Label is at the last entry of stack then the value is set to one else is set to zero. The last one is the (TTL) value. TTL value decreases by one on every hop as it passes through the LSRs. The packet is dropped when the TTL value reaches zero. Among all these fields of MPLS shim header, label plays a very important role [11][17][18].

\subsection{MPLS Elements}

- Label: it serves to identify the path that the packet must follow in the MPLS network which permits the routers to increase the routing speed.

- Label Switch Router (LSR): A router which is located in the MPLS domain and forwards the packets based on label switching is called LSR and usually this type is located the provider cloud; as soon as LSR receives a packet it checks the lookup table and determines the next hop, then before forwarding the packet to next hop it removes the old label from the header and attaches new label.

- $\quad$ Label Edge Router (LER): LER handles L3 lookups that is responsible for adding or removing the labels from the packets when they enter or leave the MPLS domain. Whenever a packet is entering or leaving 
MPLS domain it has to pass through LER router, when a packet enters into MPLS domain through LER which is called "Ingress router", or when a packet leaves the MPLS domain through LER which is called Egress router.

- Label Distribution Protocol (LDP): the protocol where the label mapping information is exchanged between LSRs. It is responsible for establishing and maintaining labels between switches and routers.

- Forward Equivalence Class (FEC): set of packets where they have related characteristics which are forwarded with the same priority to the same path. This set of packets is has the same MPLS label. Each packet in MPLS network is assigned with FEC only once at the Ingress router.

- $\quad$ Label Switched path (LSP): the path set by signaling protocols in MPLS domain. In MPLS domain there are number of LSPs that are originated at ingress router and traverses one or more core LSRs and terminates at egress router. There are two ways to create LSPs in the MPLS network, one is control driven LSP and the other is explicitly routed LSP. Control driven LSP are also called hopby-hop LSP which are set using LDP protocol. Explicitly routed LSPs are also called constraint based routed LSPs (CRLSPs) [5][9][11] [15][16], and that's will be used in our simulation to define the path.

MPLS node has two planes:

Control Plane: It is responsible for the routing information exchange and label distribution between adjacent devices.

Data Plane: It is responsible for forwarding packets according to the destination IP address or label using LFIB managed by the control plane.

In MPLS routers control plane and data plane are separated entities. This separation allows the deployment of a single algorithm that is used for multiple services and traffic types [7][19].

\section{TRAFFIC ENGINEERING IN MPLS NETWORKS}

The modern networks are converged networks; they carry voice, video and normal data by using the same network resources. Since some user data traffics such as voice, video or SQL bank

Transactions are more important and less tolerant to delay; they are preferentially treated based on their delivery requirements such as bandwidth and maximum affordable delay. Considering the increased number of internet users and different network data traffic types, internet service providers (ISP) face a challenge in the form of Traffic Engineering [20]. The provision of traffic engineering through conventional IP networks is really a challenging task. In this type of networks, IP packets are forwarded while considering the Open shortest path first (OSPF) protocol which chooses the shortest path from source to destination. Although the selection of the shortest paths may save network resources, however they may lead to problems [11]. To tackle the problem of low delay and packet loss during the delivery of multimedia applications, it is necessary to think of improvement methods to use more effectively the available network resources. MPLS-TE are some process that provides this functionality [9]. Although the original idea behind the development of MPLS was to facilitate fast packet switching, currently its main goal is to support traffic engineering and provide quality of service [14]. Traffic engineering is mainly needed when the goal is to achieve the performance objectives such as traffic placement on specific links and optimization of network resources. This indicates considering a path between source and destination with different constraints, and also forwarding traffic along this specific path. However, forwarding traffic through this path is not achievable with IP, because the IP forwarding decision at each hop is based on the destination address, which is often made independently. The routing capabilities of MPLS let the LSP source to calculate the path, build MPLS forwarding state, and map the packets into that particular LSP. Upon mapping the packet, the forwarding step is done depending on the label, where all of the intermediate hops are able to make independent decision to forward the packets. The concept of traffic trunk has been established in order to implement TE in a MPLS domain. A traffic trunk is defined as a collection of traffic flows located inside an LSP [21][22]. 
4

\section{SIMULATION METHODOLOGY}

We have used OPNET Modeler 14.0 to create the topology as shown in Fig.5 and Fig. 6 for both traditional and MPLS networks. The simulations consist of two scenarios with considering the same network topology. Scenario 1 on the basis of IP network without TE (composed of OSPF). Scenario 2 on the basis of MPLS network with TE. The results from these simulations are used for comparison between the two networks.

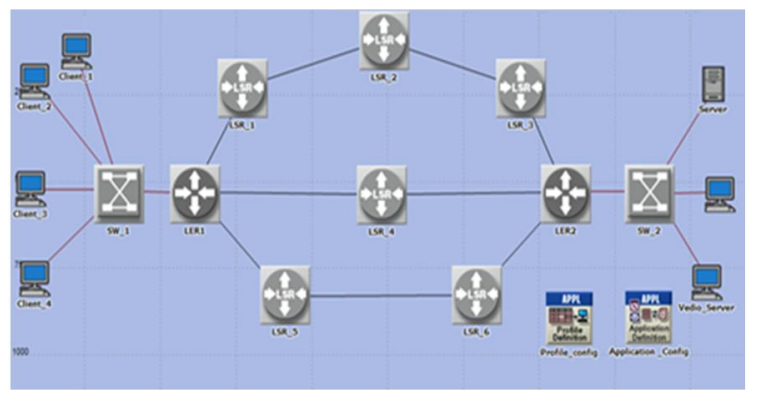

Fig. 5 (Scenario 1)

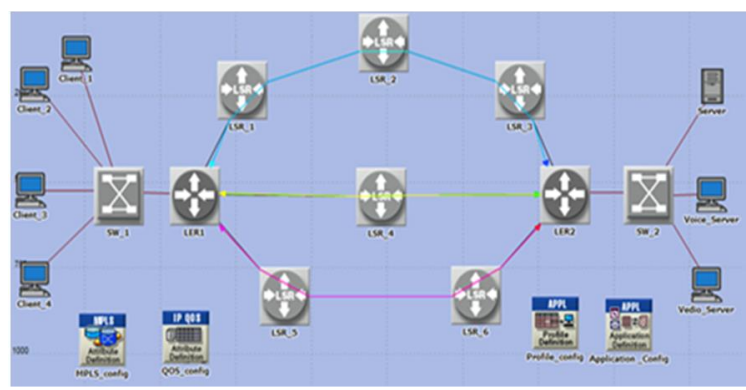

Fig. 6 (Scenario 2)

The network consists of the following elements: Two LERs (Ingress LER1and Egress LER2), six LSRs (LSR_1, LSR_2, LSR_3, LSR_4, LSR_5 and LSR 6) these routers are interconnected via PPP adv link operate at $4.5 \mathrm{Mbps}$ data rate, two switches (SW1 and SW2), links used to connect switches with routers (100BaseT), while 10BaseT is to connect the workstations with the switches, all the links used are Full duplex links. Four clients (client_1, client_2, client_3, client_4) and three servers FTP server, voice server, video server) are used. Each client use three type of traffic: VoIP, video conferencing and file transfer protocol FTP.

\subsection{Assumption}

For each scenario the duration of the simulation is 400 seconds. The traffic starts at the 110th second and ends at the 400th second of the simulation time. One of the key factors of this experiment is that it considers different network loads in the two scenarios because without having congestion in the network TE will not be needed. In the context of network load, the importance of varying network would be realized while configuring and simulating the network models. For example, a medium network load, high network load then a worst possible network load can be considered in order to understand the impact on the performance of different performance metrics. The network topology

Scenarios considered are partially meshed on the implication with a small ISP domain as because of partially meshed topology would be an ideal choice of IP and MPLS domain corresponded to a realistic network topology. The FTP, video and voice traffic is generated in a way that each flow of the traffic can be aggregated. Under the worst possible network load, the models would strongly be focused while analyzing the obtained results.

\section{SIMULATION AND RESULTS}

We have compared performance metric of MPLS_TE and IP model networks. The compared parameters were End-to-End Delay, Delay Variation, Packet Send and Receive, FTP Response Time. For all the above parameters, MPLS TE performance was better than traditional IP network model. In the case of worst possible network load (heavy load), the better performance for MPLS_TE is more apparent.

In Scenario1 (Fig.5) all routers are normal IP routers. MPLS definition attribute, is not considered and the packets are routed using OSPF protocol, therefore all packets are transmitted through the shortest path only (LER $1<->$ LSR $4<-$ $>$ LER2) and doesn't consider the other two paths. Since In Scenario2 (Fig. 6) MPLS_TE is implemented by creating LSPs, and defining how traffic is assigned to the corresponding LSPs. Before LSPs are configured, status of MPLS on the Interfaces running OSPF of core routers is set to enable. The edge routers, LER1 and LER2 are considered as the source and destination of the LSPs, respectively. In order to make LSPs reachable from other sections of the MPLS domain, a loopback interface on the routers has been configured. The network load is evenly distributed between the tree LSPs :(LER $1<->$ LSR_ $1<-$ $>$ LSR_2<->LSR_3,LER1<->LSR_4<->LER2 and LER1 $<->$ LSR_5<->LSR_6<->LER2) these makes MPLS an efficient technology. 
A. Sulaiman and O. Alhafidh / International Journal of Computer Networks and Communications Security, 2 (3), March 2014

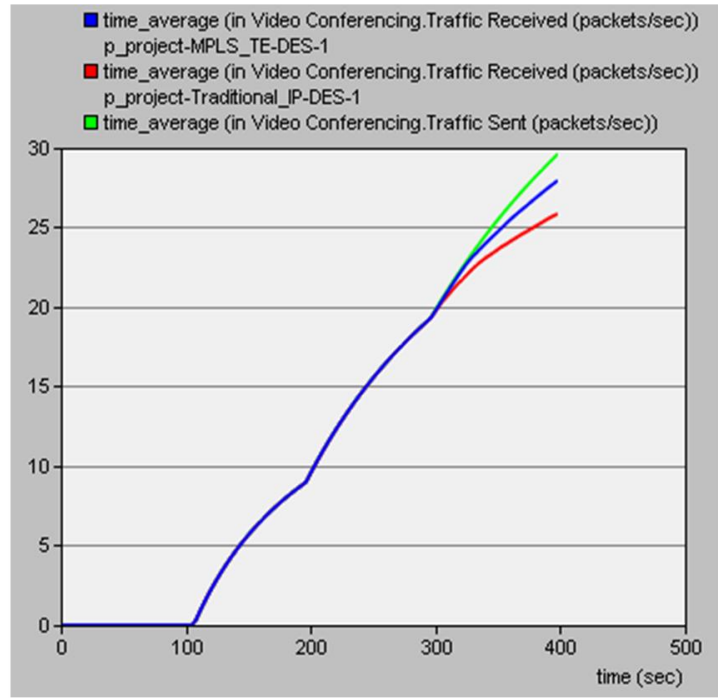

Fig. 7 Video Packet Send and Received

Fig.7 and Fig. 8 give the average number of packets sent and received in both MPLS and conventional IP networks for both voice and video traffic. Simulation result shows that MPLS model gives more throughput than the IP model, and shows that in the IP network Video and voice packets start to drop earlier than in MPLS network when worst possible network load. MPLS delivers the packets with high transmission speed and lower delays. There is TE implemented in the MPLS network which temporarily reduces the congestion. Due to these factors the packet drop in MPLS network is lower than IP network; this increases the throughput in the MPLS network.

The end to end delay of voice and video traffics are given in Fig. 9 and Fig 10. It is clear that MPLS had lower delay than the IP model in the case of worst network load (heavy). Delay in the case of both interactive voice is less than $200 \mathrm{~ms}$ and in video is less than $250 \mathrm{~ms}$. The jitter or delay variation was around (30-50) $\mathrm{ms}$ as per standard in ITU [1][3][23].

The delay variation of voice and video traffics are given in Fig.11 and Fig. 12, Similar to the end to end delay results, the delay variation result shows that MPLS TE had lower delay than IP network model in the case of heavy load, and the delay variation exceed the threshold in IP model earlier than MPLS TE. From the results in Fig.13 we note that Voice Packet Jitter increased in both network model but in MPLS TE still much lower than IP network model.

The FTP response time of MPLS TE was much lower than IP network model as in Fig. 14, also the average of packet received in MPLS TE better than IP network model as in Fig. 15.

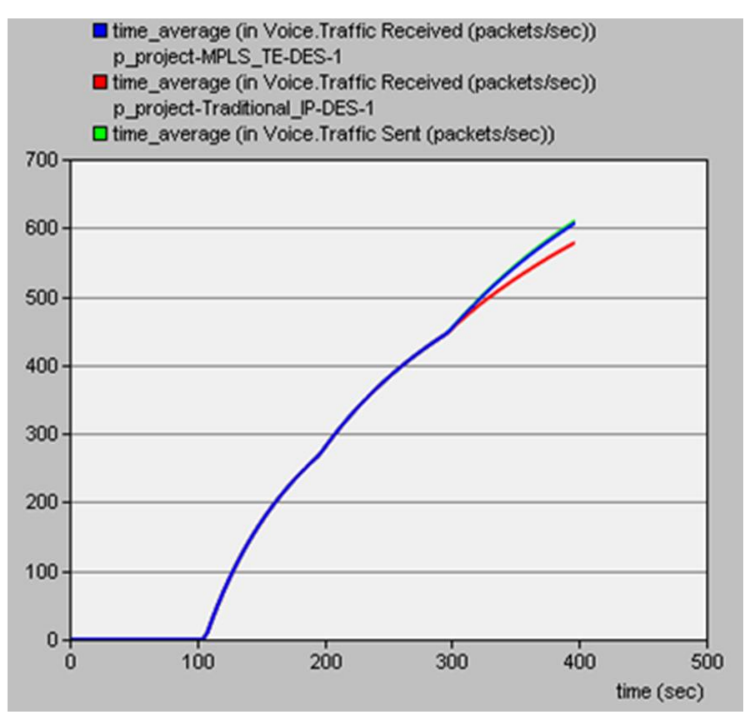

Fig. 8 Voice Packet Send and Received

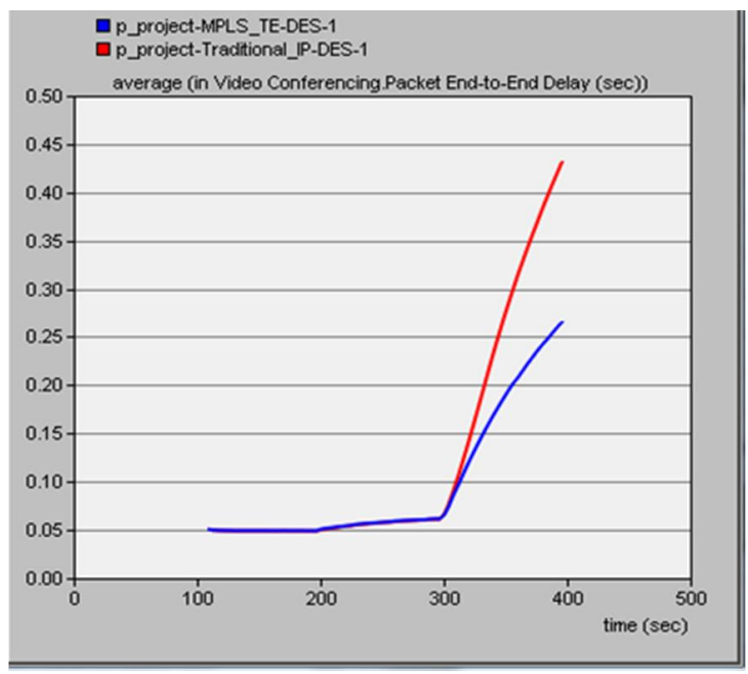

Fig. 9 Video Packet End to End Delay

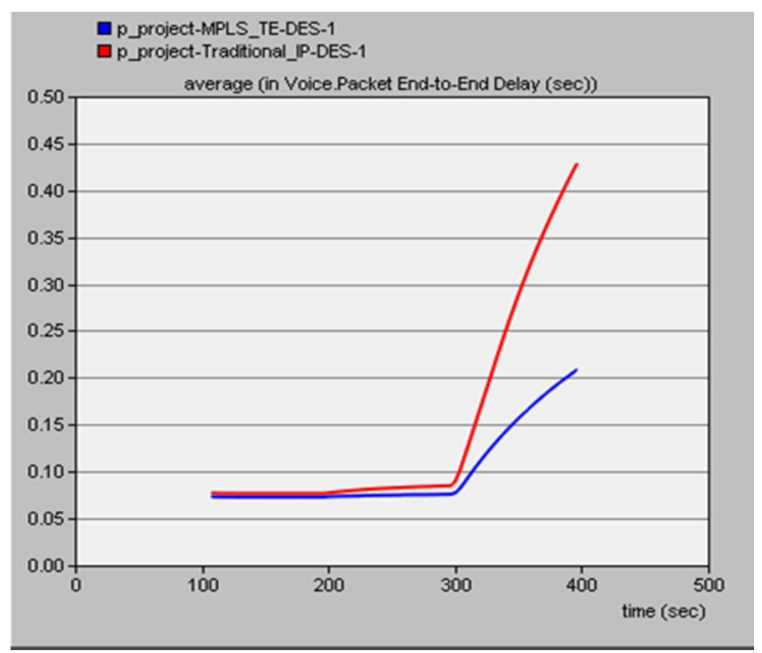

Fig. 10 Voice Packet End to End Delay 
A. Sulaiman and O. Alhafidh / International Journal of Computer Networks and Communications Security, 2 (3), March 2014

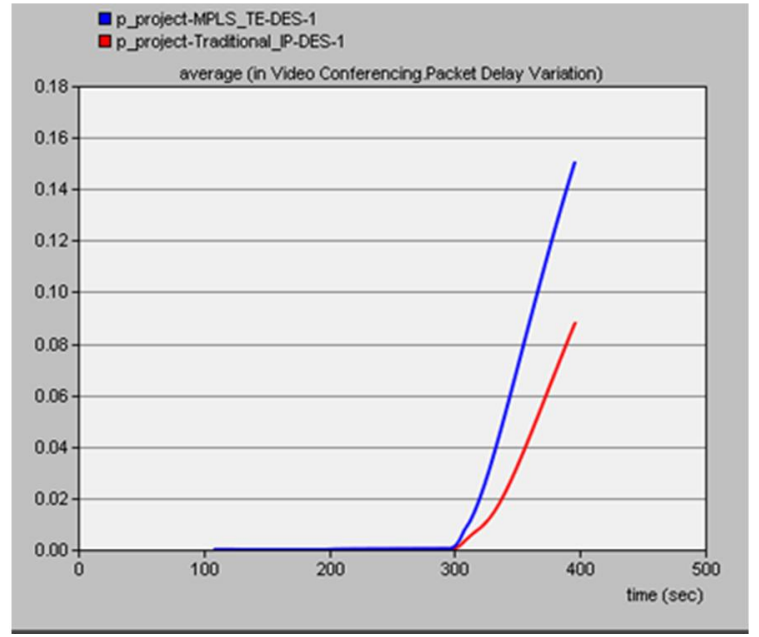

Fig. 11 Video Packet Delay Variation

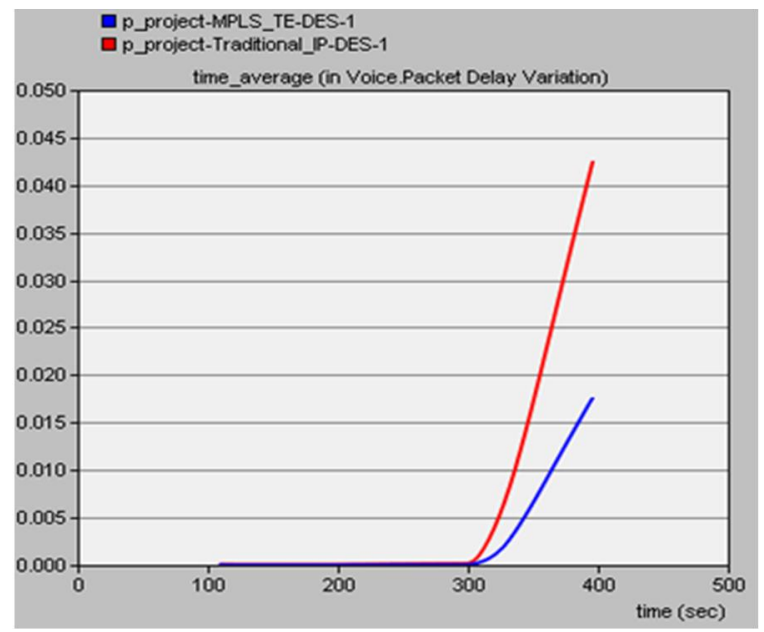

Fig. 12 Voice Packet Delay Variation

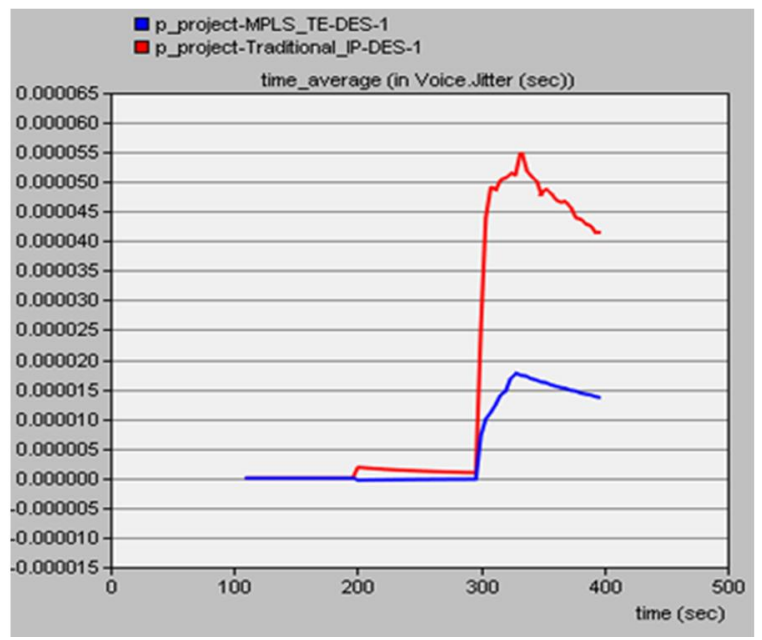

Fig. 13 Voice Packet Jitter

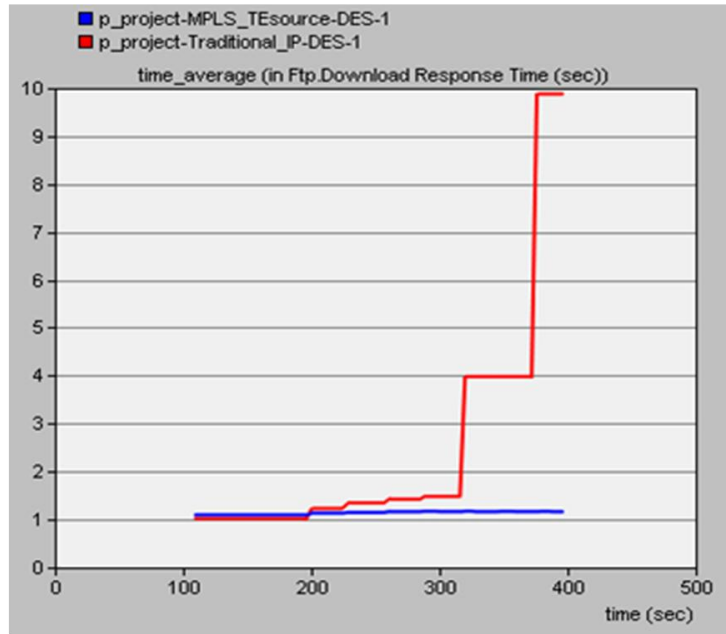

Fig. 14 FTP Download Response Time

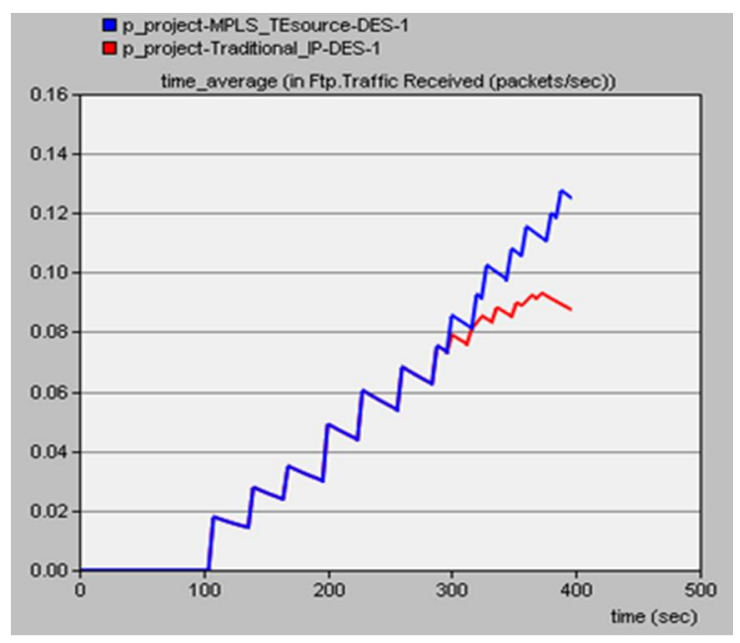

Fig. 15 FTP Traffic Received

\section{CONCLUSION}

The main objective of the paper is based on the performance analysis of conventional IP network and MPLS TE network in respect of multimedia applications (VoIP, Video Conferencing) and Non Real Time application (FTP). Based on the simulation results it can be concluded that MPLSTE provides best solution in implementing such applications compared to conventional IP networks. Also this paper explains poor link utilization in conventional IP networks. It is seen that network configured with OSPF routing techniques are not capable of handling the incoming traffic efficiently. When the network traffic increases, shortest path from source node to destination node is heavily congested and lead to loss of transmission data. 
We have simulated and shown the MPLS TE is capable of handling incoming traffic efficiently by distributing the traffic over several LSPs according to FEC (Forwarding Equivalence Class) which is not able to achieve in conventional routing protocol. Through the results analysis, it is clear that with proper MPLS TE applied to the network, the performance of the network is significantly improved. Therefore, Internet service providers and network providers seem to have been taking the advantage of this technology to provide flexible support for a wide range of services, simplify network architecture, build reliable internet services and overcome some existing infrastructure limitations; also we propose to implement this technology in the Mosul University Network in Iraq .as a future work.

\section{REFERENCES}

[1] Yan Chen, Toni Farley and Nong Ye, "QoS Requirements of Network Applications on the Internet", International Journal of Information Knowledge Systems Management, 2004, IOS Press (55-76).

[2] Er. Sourabh Jain. July, "Performance Analysis of Voice over Multiprotocol Label Switching Communication Networks with Traffic Engineering", International Journal of Advanced Research in Computer Science and Software Engineering, Volume 2, Issue 7, 2012.

[3] Junaid Ahmed Zubairi., "Voice Transport Techniques over MPLS", International Symposium of High Capacity Optical Networks and Enabling Technologies, 2008.

[4] Aruna Kapoor, Sunila Godara, Sanjeev Khambra, Amandeep, "Comparative Analysis of Signaling Protocols in Mpls Traffic Engineering", National Workshop-CumConference on Recent Trends in Mathematics and Computing (RTMC), 2011.

[5] Dr. Reyadh Shaker Naoum and Mohanand Maswady, "Performance Evaluation for VOIP over IP and MPLS", World of Computer Science and Information Technology Journal (WCSIT), Vol. 2, No. 3, 2012, 110-114.

[6] Jasmina Barakovic Himzo Bajric, and Amir Husic., "Multimedia Traffic Analysis of MPLS and non-MPLS Network", International Symposium ELMAR-2006, Zadar, Croatia, June 2006.

[7] Mahesh Kr. Porwal, Anjulata Yadav, S. V. Charhate. July, "Traffic Analysis of MPLS and Non MPLS Network including MPLS Signaling Protocols and Traffic distribution in
OSPF and MPLS", International Conference on Emerging Trends in Engineering and Technology, ICETET, June 2008.

[8] Md. Arifur Rahman, Ahmedul Haque Kabir, K. A. M. Lutfullah, "Performance Analysis and the Study of the behavior of MPLS Protocols", International Conference on Computer and Communication Engineering, Kuala Lumpur, Malaysia, May 2008.

[9] Abdellah Jamali, Najib Naja and Driss El Ouadghiri, "An Enhanced MPLS-TE for Transferring Multimedia packets", International Journal of Advanced Computer Science and Applications, (IJACSA), Vol. 3, No.8, 2012.

[10] Keerthi P. Jannu. June, “OPNET simulation of voice over MPLS with Considering Traffic Engineering", M.Sc. Thesis, School of Computing, Blekinge Institute of Technology, Sweden, 2010.

[11]Faiz Ahmed and Dr. Irfan Zafar, "Analysis of traffic engineering parameters while using multi-protocol label switching (MPLS) and traditional IP networks", Asian Transactions on Engineering (ATE ISSN: 2221-4267) Volume 01 Issue 03, 2011.

[12] Anupkumar M Bongale and Nithin N., "Analysis of Link Utilization in MPLS Enabled Network using OPNET IT Guru", International Journal of Computer Applications (0975 - 8887), Volume 41- No.14, March 2012.

[13] E. Rosen, A. Viswanathan, R. Callon. January, "Multiprotocol Label Switching Architecture", Network Working Group RFC 3031, 2001.

[14] Wei Sun Praveen Bhaniramka, Raj Jain, "Quality of Service using Traffic Engineering over MPLS: An Analysis", 25th Annual IEEE Conference, Local Computer Networks, LCN Proceeding, 2000.

[15] Abdellah Jamali, Najib Naja, Driss EI Ouadghiri and Redouane Benaini, "Improving Quality of Service (QoS) in Multi-Protocol Label Switching Module”, IEEE Mediterranean Microwave Symposium, Nov 2009.

[16] Hang Man and Yang Li, "Multi-Stream Video Transport Over MPLS Networks", IEEE Workshop of Multimedia Signal Processing, Dec 2002.

[17] Jeevan Kharel, "Performance Evaluation of Voice Traffic over MPLS Network with TE and QoS Implementation", M.Sc. thesis, School of Computing Blekinge Institute of Technology, Sweden, Nov 2011. 
[18] Muhammad R. A. Rahimi, Habibah Hashim, Ruhani $\mathrm{Ab}$ Rahman, "Implementation of Quality of Service (QoS) in Multi-Protocol Label Switching (MPLS) Networks", International Colloquium on Signal Processing \& Its Applications (CSPA), 2009.

[19] Jawad Oubaha, Adel Echchaachoui, Ali Ouacha, and Mohammed Elkoutbi, "New Method: Mapping of 802.11e into MPLS Domains, Conception and Experimentation", Springer-Verlag Berlin Heidelberg CCIS 189, 2011, pp. 470-483.

[20] Mohsin Khan, "MPLS Traffic Engineering in ISP Network", International Journal of Computer Applications (0975 - 8887), Volume 59- No.4, Dec 2012.

[21]Tatiana Onali, "Quality of Service Technologies for Multimedia Applications in Next Generation Networks", Ph.D. thesis, University of Cagliari, Italy, 2009.

[22]H. H. Jonathan Chao, Xiaolei Guo, "Quality of Service Control In High-Speed Networks" ,Copyright@2002 John Wiley \& Sons, Inc, 2002.

[23] James F. Kurose, Keith W. Ross, "Computer Networking: A Top Down Approach Featuring the Internet", 6th edition. University of Massachusetts. Published by Pearson, 2011.

[24]E. Landivar, "Unified communications with Elastix", e-book, 2nd Edition, ASIN: B0056IXJRC,2011, available at[http://www.elastix.org/index.php/en/product -information/manuals-ooks.html]. 